\title{
REDUCED P.P.-RINGS WITHOUT IDENTITY
}

\author{
XIAOJIANG GUO AND K. P. SHUM
}

Received 16 March 2006; Accepted 19 March 2006

We give some necessary and sufficient conditions for p.p.-rings without identity to be reduced. Our results strengthen and extend the results of Fraser and Nicholson as well as some recent results we obtained on reduced p.p.-rings with identity.

Copyright (c) 2006 Hindawi Publishing Corporation. All rights reserved.

\section{Introduction}

Throughout this paper, the ring $R$ is not necessarily with an identity. We denote the set of all idempotents of $R$ by $E(R)$. Also, for a subset $X \subseteq R$, we denote the right (resp., left) annihilator of $X$ in $R$ by $\operatorname{ann}_{r}(X)$ (resp., $\operatorname{ann}_{\ell}(X)$ ).

Now, according to Fraser and Nicholson in [5], we call a ring $R$ a left p.p.-ring, in brevity, l.p.p.-ring, if for all $x \in R$, there exists an idempotent $e$ such that $\operatorname{ann}_{\ell}(x)=$ $\operatorname{ann}_{\ell}(e)$ and $e x=x$. Dually, we may define a right p.p.-ring. Naturally, we call a ring $R$ a p.p.-ring if it is both an l.p.p.-ring and an r.p.p.-ring. Clearly, if the ring $R$ has an identity, the above (left; right) p.p.-rings coincide with the (left; right) p.p.-rings discussed in [6]. It can be observed that the class of p.p.-rings contains the classes of regular (von Neumann) rings, hereditary rings, Baer rings, and semi-hereditary rings as its proper subclasses. In the literature, p.p.-rings have already been studied by many authors (see $[1,2,5-9,11])$. It is noteworthy that the definition of p.p.-rings has been extended to semigroups; in particular, Fountain [4] has introduced the concept of abundant semigroups which are both l.p.p.- and r.p.p.- semigroups. Similar to p.p.-rings, the class of abundant semigroups contains the class of regular semigroups as its proper subclass. An r.p.p.-semigroup in which every idempotent is central is called a $C$-r.p.p.-semigroup. In 1977 Fountain [3] first proved that a $C$-r.p.p.-monoid can be expressed as a strong semilattice of left cancellative monoids. This shows that a $C$-r.p.p.-monoid does not contain any nonzero nilpotent element and hence it is a reduced semigroup.

On the other hand, Cornish and Stewart [2] called a ring $R$ reduced if it contains no nonzero nilpotent elements. Obviously, the left annihilator $\operatorname{ann}_{\ell}(X)$ of $X$ in a reduced $\operatorname{ring} R$ is always a two-sided ideal of $R$. Moreover, if $R$ is a reduced ring, then $e f=0$ if and 
only if $f e=0$ for any nonzero idempotents $e, f \in R$. By using the concept of a reduced ring, Fraser and Nicholson [5] obtained an analogous result of Fountain's [4] that a ring $R$ is a reduced p.p.-ring if and only if $R$ is a (left; right) p.p.-ring in which every idempotent is central.

In view of the above result, one would naturally ask whether we can give some necessary and sufficient conditions for a p.p.-ring to be reduced. In this aspect, the authors [6] have recently given some characterization theorems for reduced p.p.-rings with identity. In this paper, we further investigate this question for p.p.-rings without identity by using the properties of abundant semigroups. Some new characterization theorems for a p.p.ring without identity to be reduced are obtained. Our results strengthen and extend the results obtained by Fraser and Nicholson [5] and those by the authors in [6]. The concept of perpetual ideals in p.p.-rings is also introduced.

\section{Preliminaries}

We first cite some known results of abundant semigroups which will be used in the sequel.

Let $\mathscr{L}^{*}$ and $\mathscr{R}^{*}$ be the left and right Green starred relations on an abundant semigroup $S$, as described by Fountain in $[3,4]$. Then we have the following lemma.

Lemma 2.1 [4]. Let $S$ be a semigroup. Then, for any elements $a, b \in S, a \mathscr{L}^{*} b\left[a \mathscr{R}^{*} b\right]$ if and only if $a x=a y \Leftrightarrow b x=b y[x a=y a \Leftrightarrow x b=y b]$, for all $x, y \in S^{1}$, where $S^{1}$ is the semigroup $S$ adjoined with an identity 1 .

As an easy but useful consequence, we have the following corollary.

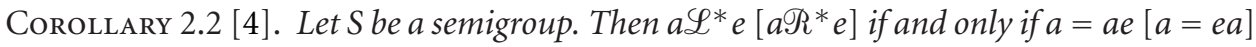
and $a x=a y \Rightarrow e x=e y[x a=y a \Rightarrow x e=y e]$, for all idempotents $e \in S$ and $x, y \in S^{1}$.

In view of the above corollary, we call an element $a \in S$ a right abundant element if there exists an idempotent $e \in E(S)$ such that $e \mathscr{L}^{*} a$. Dually, we call an element $a \in S$ a left abundant element if there exists an idempotent $f \in E(S)$ such that $f \Re^{*} a$. An element is called abundant if it is both left and right abundant. A semigroup $S$ is called abundant if every element of $S$ is abundant.

The following lemma was due to Lawson [10].

Lemma 2.3 [10]. Let $S$ be an abundant semigroup and $e^{2}=e \in S$. Then eSe is an abundant subsemigroup of $S$.

LEMMA 2.4. The following statements hold on a ring $R$.

(1) If $R$ has an identity, then $R$ is a p.p.-ring if and only if the multiplicative semigroup $(R, \bullet)$ of $R$ is an abundant semigroup.

(2) If $R$ is a p.p.-ring, then the multiplicative semigroup $(R, \bullet)$ is an abundant semigroup.

Proof. (1) This part is trivial. (2) Assume that $R$ is a p.p.-ring. Let $a \in R$ and $u, v \in(R, \bullet)^{1}$ with $u a=v a$. Then because $R$ is a p.p.-ring, there exists $e \in E(R)$ such that $\operatorname{ann}_{\ell}(a)=$ $\operatorname{ann}_{\ell}(e)$ and $e a=a$. We consider the following two cases. 
(i) $u, v \in R$. Then $(u-v) a=0$ and so $(u-v) e=0$, that is, $u e=v e$.

(ii) One of $u$ and $v$ does not lie in $R$; say $v \notin(R, \bullet)$. Then, in this case, $v=1$ and hence we have $u a=a$. Since $e a=a$, we have $(u-e) a=0$ and thereby, $(u-e) \in$ $\operatorname{ann}_{\ell} a=\operatorname{ann}_{\ell} e$, that is, $u e=e$.

Thus $a \mathscr{R}^{*} e$ and hence $(R, \bullet)$ is a left abundant semigroup. Dually, we can similarly prove that $(R, \bullet)$ is a right abundant semigroup. Therefore $(R, \bullet)$ is an abundant semigroup.

It is well known that the Green starred relations $\mathscr{L}^{*}$ and $\mathscr{R}^{*}$ are congruences on an abundant semigroup $S$. Now, following Fountain [4], we define $\mathscr{H}^{*}$ as the intersection of the congruences $\mathscr{L}^{*}$ and $\mathscr{R}^{*}$ on a semigroup $S$. Then, we call a semigroup $S$ a superabundant semigroup if each $\mathscr{H}^{*}$-class of $S$ contains an idempotent in $S$. The structure of superabundant semigroups has recently been studied by Ren and Shum in [12]. Of course, a superabundant semigroup is always abundant.

By a band we mean a semigroup $B$ whose elements are idempotents. In addition, if a band $B$ is commutative, then we call $B$ a semilattice.

As in [6], we now denote the $2 \times 2$ upper triangular matrix rings over $\mathbb{Z}$ and $\mathbb{Z}_{p}$ by $\mathrm{UTM}_{2}(\mathbb{Z})$ and $\mathrm{UTM}_{2}\left(\mathbb{Z}_{p}\right)$, respectively.

The following result for reduced p.p.-rings with identity was proved in [6].

Lemma 2.5 [6]. Let $R$ be a p.p.-ring with an identity. Then $R$ is reduced if and only if $R$ has no subrings which are isomorphic either to $U T M_{2}(\mathbb{Z})$ or to $U T M_{2}\left(\mathbb{Z}_{p}\right)$, where $p$ is a prime.

\section{Characterization theorems}

By using the results cited in the above section, we now establish some new characterization theorems for reduced p.p.-rings possibly without an identity.

THEOREM 3.1. The following statements are equivalent for a p.p.-ring $R$ :

(1) $R$ is reduced;

(2) $E(R)$ is central in $R$;

(3) $(E(R), \bullet)$ is a semilattice;

(4) $(E(R), \bullet)$ is a band;

(5) for all $e, f \in E(R), e f=0$, if and only if $f e=0$.

Proof. We only need to prove that $(1) \Rightarrow(2)$ and $(2) \Rightarrow(1)$. This is because that $(2) \Rightarrow(3) \Rightarrow(4)$ $\Rightarrow(5) \Rightarrow(2)$ is easy to see.

For $(1) \Rightarrow(2)$, we suppose that the $\operatorname{ring} R$ is reduced. Then $e x=$ exe since $(e x-e x e)^{2}=$ 0 , for all $e \in E(R)$ and $x \in R$. Similarly, $x e=e x e$. Thus $e x=x e$, for all $e \in E(R)$ and $x \in R$. That is, (2) holds.

For $(2) \Rightarrow(1)$, we suppose that every element of $E(R)$ is central in $R$. In order to prove (1), it suffices to prove that for all $x \in R, x^{2}=0$ implies that $x=0$. Since $(R, \bullet)$ is an abundant semigroup, there exists $e \in E(R)$ such that $e \mathscr{L}^{*} x$, and hence $x e=x$ by Corollary 2.2 . Now, by Lemma 2.1, we see that $x^{2}=0$ implies that $e x=0$, hence by (2), $x e=x=0$.

Theorem 3.2. Let $R$ be a p.p.-ring. Then $R$ is a reduced ring if and only if $(R, \bullet)$ is a superabundant semigroup. 
Proof

Necessity. If $R$ is reduced, then $E(R)$ is central in $R$. By a result of Fountain in [3], $(R, \bullet)$ is a strong semilattice of cancellative monoids and by a result of Fountain in $[4],(R, \bullet)$ is a superabundant semigroup.

Sufficiency. Suppose that $(R, \bullet)$ is a superabundant semigroup. To verify that $R$ is reduced, we need only to prove that $x^{2}=0$ implies that $x=0$ for all $x \in R$. Because $(R, \bullet)$ is a superabundant semigroup, there exists a unique idempotent $e \in E(R)$ such that $x^{\mathscr{H}} * e$. Hence, we have $e x=x$ by Corollary 2.2. Now, by Lemma 2.1, $x^{2}=0$ implies that $e x=0$, and so we have $x=e x=0$, as required.

We now introduce a new definition.

Definition 3.3. A ring $R$ is said to be locally reduced if the subring $e$ Re of $R$ is reduced for every $e \in E(R) \backslash\{1\}$.

Clearly, a locally reduced ring is not necessarily reduced. For example, if we consider the matrix ring

$$
M_{2}(\mathbb{Z})=:\left\{\left(\begin{array}{ll}
x & y \\
0 & 0
\end{array}\right): x, y \in \mathbb{Z}\right\},
$$

then $M_{2}(\mathbb{Z})$ is not reduced because $\left(\begin{array}{ll}0 & 1 \\ 0 & 0\end{array}\right)^{2}=\left(\begin{array}{ll}0 & 0 \\ 0 & 0\end{array}\right)$. On the other hand, if the nontrivial idempotents in $E(R) \backslash\{1\}$ are $e_{x}=\left(\begin{array}{cc}1 & x \\ 0 & 0\end{array}\right)$, then

$$
\left(\begin{array}{ll}
1 & x \\
0 & 0
\end{array}\right)\left(\begin{array}{ll}
a & b \\
0 & 0
\end{array}\right)\left(\begin{array}{ll}
1 & x \\
0 & 0
\end{array}\right)=\left(\begin{array}{cc}
a & a x \\
0 & 0
\end{array}\right) .
$$

Now, since

$$
\left(\begin{array}{cc}
a & a x \\
0 & 0
\end{array}\right)^{2}=\left(\begin{array}{cc}
a^{2} & a^{2} x \\
0 & 0
\end{array}\right)=0 \Longleftrightarrow a^{2}=0 \Longleftrightarrow a=0,
$$

we can see that every subring $e$ Re of $R$ is a reduced ring, and hence the ring $R$ itself is locally reduced.

However, if the p.p.-ring $R$ does not have an identity, then a locally reduced ring and a reduced ring are the same, as the following theorem shows.

We now give the following interesting theorem.

Theorem 3.4. Let $R$ be a p.p.-ring without identity. Then $R$ is a reduced ring if and only if $R$ is a locally reduced ring.

Proof. Obviously, a reduced ring is locally reduced. To prove the converse part, we suppose that the ring $R$ is locally reduced, that is, $e$ Re is a reduced ring for all $e \in E(R)$. Then, we can let $e, f \in E(R)$ such that $e f=0$. Denote $g=e-f e$. It is now easy to check from the fact $e f=0$ that $g \in E(R)$ and $f g=g f=0$. This leads to $u=f+g \in E(R)$. By using $e f=0$ and $f g=0$ again, we have $e u=e=u e$ and $f u=f=u f$. That is, $e, f \in u R u$. On the other hand, by Lemma 2.3, $(u R u, \bullet)$ is an abundant semigroup. Since the ring $u R u$ is reduced, $f e=0$. Thus, by Theorem $3.1, R$ is a reduced ring. 
The following corollary is a generalized version of the main result in [6].

Corollary 3.5. Let $R$ be a p.p.-ring without identity. Then $R$ is a reduced ring if and only if $R$ has no subrings which are isomorphic either to $U T M_{2}(\mathbb{Z})$ or to $U T M_{2}\left(\mathbb{Z}_{p}\right)$, where $p$ is a prime.

\section{Perpetual ideals and reduced p.p.-rings}

In this section, we define the (left; right) perpetual ideal and we characterize the reduced p.p.-rings by using the (right; left) perpetual ideals.

Definition 4.1. Let $R$ be a ring and $I$ a right ideal of $R$. Then, $I$ is called a right perpetual ideal of $R$ if for all $x \in I$, the set $\left\{r \in R: \operatorname{ann}_{\ell}(x) \subseteq \operatorname{ann}_{\ell}(r)\right.$ and $u x=x \Rightarrow u r=r$ for all $u \in$ $R\}$ is contained in $I$.

Evidently, every ring $R$ has two right perpetual ideals $\{0\}$ and $R$. Dually, we can also define left perpetual ideals. By a perpetual ideal of $R$, we mean an ideal of $R$ which is a both left and right perpetual ideal. It is easy to see that the intersection of left (right) perpetual ideals of $R$ is still a left (right) perpetual ideal of $R$. Consequently, there exists the smallest (left; right) perpetual ideal of $R$ containing $X$ for all $X \subseteq R$. We usually call this smallest (left; right) perpetual ideal of $R$ the (left; right) perpetual ideal generated by $X$ and denote it by $\left(L^{*}(X) ; R^{*}(X)\right) J^{*}(X)$. If $X=a$, then we write $L^{*}(X)=L^{*}(a), R^{*}(X)=R^{*}(a)$ and $J^{*}(X)=J^{*}(a)$. Also, we simply call the (left; right) perpetual ideal generated by $a$ the principal (left; right) perpetual ideal generated by a.

The following proposition describes the construction of $R^{*}(a)$ by $a \in R$.

Lemma 4.2. Let $R$ be a ring with identity. Then $R^{*}(x)=\operatorname{ann}_{r}\left(\operatorname{ann}_{\ell}(x)\right)$, for any $x \in R$.

Proof. We can easily check that $\operatorname{ann}_{r}\left(\operatorname{ann}_{\ell}(x)\right)$ is a right ideal of $R$. Now let $u \in R$ and $a \in \operatorname{ann}_{r}\left(\operatorname{ann}_{\ell}(x)\right)$ such that $\operatorname{ann}_{\ell}(a) \subseteq \operatorname{ann}_{\ell}(u)$. Then, since $\left(\operatorname{ann}_{\ell}(x)\right) a=0$, we have $\left(\operatorname{ann}_{\ell}(x)\right) u=0$, that is, $u \in \operatorname{ann}_{r}\left(\operatorname{ann}_{\ell}(x)\right)$. Now, we have

$$
u x=x \Longleftrightarrow(u-1) x=0 \Longleftrightarrow u-1 \in \operatorname{ann}_{\ell}(x)
$$

Therefore, $\operatorname{ann}_{r}\left(\operatorname{ann}_{\ell}(x)\right)$ is indeed a right perpetual ideal of $R$. Now let $I$ be a right perpetual ideal of $R$ containing $x$. Then $\left\{u \in R: \operatorname{ann}_{\ell}(x) \subseteq \operatorname{ann}_{\ell}(u)\right\} \subseteq I$. Since $\operatorname{ann}_{\ell}(x) \subseteq$ $\operatorname{ann}_{\ell}(u)$ if and only if $\left(\operatorname{ann}_{\ell}(x)\right) u=0$ if and only if $u \in \operatorname{ann}_{r}\left(\operatorname{ann}_{\ell}(x)\right)$, we can easily observe that $\operatorname{ann}_{r}\left(\operatorname{ann}_{\ell}(x)\right) \subseteq I$. Thus $\operatorname{ann}_{r}\left(\operatorname{ann}_{\ell}(x)\right)$ is the smallest right perpetual ideal of $R$ containing $x$, whence $R^{*}(x)=\operatorname{ann}_{r}\left(\operatorname{ann}_{\ell}(x)\right)$.

By using the above result, we obtain the following lemma.

Lemma 4.3. Let $(R,+, \bullet)$ be a ring. Now denote the semigroup $(R, \bullet)$ by $R$. Then the following statements hold:

(1) if $e \in E(R)$, then $R^{*}(e)=e R$;

(2) if $x \in R, e \in E(R)$ and $x \mathscr{R}^{*} e$, then $R^{*}(x)=e R$.

Proof. (1) We need only to show that $e R$ is a right perpetual ideal of $R$. Obviously, $e R$ is a right ideal of $R$. Now let $y \in R$ such that $\operatorname{ann}_{\ell}(x) \subseteq \operatorname{ann}_{\ell}(y)$ and $u(e x)=e x \Rightarrow u y=y$ 
for all $u \in R$. Then, since $e(e x)=e x$, we have $e y=y \in e R$. This means that $e R$ is a right perpetual ideal of $R$. Thus $R^{*}(e)=e R$.

(2) By Lemma 2.1, $e \in R^{*}(x)$ and $e R \subseteq R^{*}(x)$. On the other hand, since $e x=x$, we have $x \in e R$. But $e R$ is a right perpetual ideal of $R$ (by (1)); by the minimality of $R^{*}(x)$, we obtain that $R^{*}(x) \subseteq e R$. Thus $R^{*}(x)=e R$.

Finally, we obtain the main result of this section.

THEOREM 4.4. The following statements are equivalent for a p.p.-ring $R$ without identity:

(1) $R$ is reduced;

(2) $R$ is a locally reduced ring;

(3) every left perpetual ideal of $R$ is a right perpetual ideal of $R$;

(4) every right perpetual ideal of $R$ is a left perpetual ideal of $R$;

(5) for all $a \in R, L^{*}(a)$ is a right perpetual ideal of $R$;

(6) for all $a \in R, R^{*}(a)$ is a left perpetual ideal of $R$;

(7) for all $a \in R, L^{*}(a)=R^{*}(a)$.

Proof. We have already proved that (1) and (2) are equivalent. We only to need prove that $(1) \Rightarrow(3) \Rightarrow(5) \Rightarrow(1) \Rightarrow(7) \Rightarrow(1)$ because $(1) \Rightarrow(4) \Rightarrow(6) \Rightarrow(1) \Rightarrow(7) \Rightarrow(1)$ can be similarly proved.

$(1) \Rightarrow(3)$. Assume that the ring $R$ is reduced. Then, by Theorem 3.1, $E(R)$ is central in $R$. Let $I$ be a left perpetual ideal of $R$. Then $I=\bigcup_{a \in I} L^{*}(a)$. By Theorem 3.2, $(R, \bullet)$ is a superabundant semigroup and hence we have $e \in E(R)$ such that $a \mathscr{H}^{*} e$. Moreover, by Lemmas 2.1 and 4.3, $L^{*}(a)=R e=e R=R^{*}(a)$. Thus $I=\bigcup_{a \in I} R e=\bigcup_{a \in I} e R$ is a right ideal of $R$. By observing that $I=\bigcup_{a \in I} L^{*}(a)=\bigcup_{a \in I} R^{*}(a)$, we can easily see that $I$ is a right perpetual ideal of $R$.

$(3) \Rightarrow(5)$. This part is trivial.

$(5) \Rightarrow(1)$. Assume that (5) holds. Then for all $e \in E(R), L^{*}(e)=R e$ is a right ideal of $R$. But $e \in R e$, so we have $e R \subseteq R e$. If $f \in E(R)$, then ef $=x e=e f e$, where $x \in R$, hence $e f=(e f)^{2}$. This means that $E(R)$ is a band. By Theorem 3.1, $R$ is reduced.

$(1) \Rightarrow(7)$. Assume that $R$ is reduced. Then, by (6), $R^{*}(a)$ is a left perpetual ideal of $R$, for all $a \in R$. But $a \in R^{*}(a)$, we have $L^{*}(a) \subseteq R^{*}(a)$. Dually, we have $R^{*}(a) \subseteq L^{*}(a)$. Thus, $R^{*}(a)=L^{*}(a)$.

$(7) \Rightarrow(1)$. If (7) holds, then by Lemma 4.3 we have $R e=L^{*}(e)=R^{*}(e)=e R$, for all $e \in E(R)$. By the proof of $(5) \Rightarrow(1)$, we can prove that $R$ is indeed reduced.

\section{Acknowledgments}

This work is jointly supported by the Natural Science Foundation of Jiangxi Province, the Science Foundation of the Education Department of Jiangxi Province, and the Foundation of Jiangxi Normal University, China. The research is partially supported by a UGC (HK) Grant 2160210 (03/05).

\section{References}

[1] E. P. Armendariz, A note on extensions of Baer and p.p.-rings, Journal of the Australian Mathematical Society 18 (1974), 470-473. 
[2] W. H. Cornish and P. N. Stewart, Rings with no nilpotent elements and with the maximum condition on annihilators, Canadian Mathematical Bulletin 17 (1974), 35-38.

[3] J. B. Fountain, Right p.p.-monoids with central idempotents, Semigroup Forum 13 (1976/1977), no. $1,229-237$.

[4] _ Abundant semigroups, Proceedings of the London Mathematical Society. Third Series 44 (1982), no. 1, 103-129.

[5] J. A. Fraser and W. K. Nicholson, Reduced p.p.-rings, Mathematica Japonica 34 (1989), no. 5, $715-725$.

[6] X. Guo and K. P. Shum, On p.p.-rings which are reduced, to appear in International Journal of Mathematics and Mathematical Sciences.

[7] Y. Hirano, M. Hongan, and M. Ôhori, On right p.p.-rings, Mathematical Journal of Okayama University 24 (1982), no. 2, 99-109.

[8] C. Y. Hong, N. K. Kim, and T. K. Kwak, Ore extensions of Baer and p.p.-rings, Journal of Pure and Applied Algebra 151 (2000), no. 3, 215-226.

[9] S. Jøndrup, P.P.-rings and finitely generated flat ideals, Proceedings of the American Mathematical Society 28 (1971), 431-435.

[10] M. V. Lawson, The natural partial order on an abundant semigroup, Proceedings of the Edinburgh Mathematical Society 30 (1987), no. 2, 169-186.

[11] Z. Liu and J. Aksan, P.P.-rings of generalized power series, Acta Mathematica Sinica. New Series 16 (2000), no. 4, 573-578.

[12] X. M. Ren and K. P. Shum, The structure of superabundant semigroups, Science in China. Series A 47 (2004), no. 5, 756-771.

Xiaojiang Guo: Department of Mathematics, Jiangxi Normal University, Nanchang, Jiangxi 330027, China

E-mail address: xjguo@jxnu.edu.cn

K. P. Shum: Faculty of Science, The Chinese University of Hong Kong, Shatin, Hong Kong

E-mail address: kpshum@math.cuhk.edu.hk 


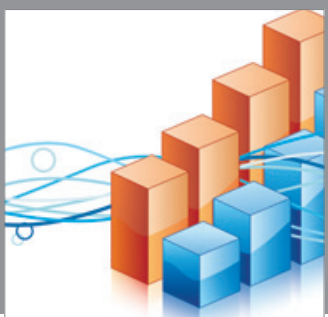

Advances in

Operations Research

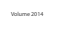

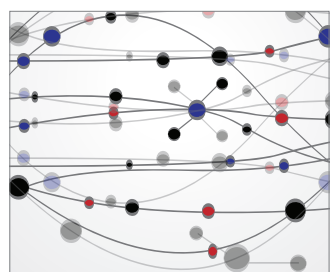

\section{The Scientific} World Journal
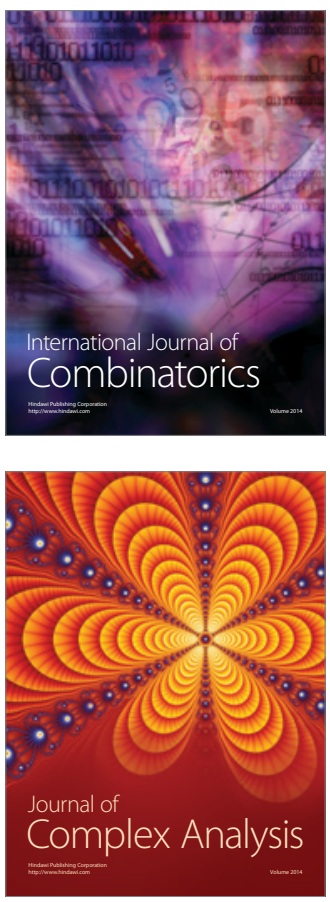

International Journal of

Mathematics and

Mathematical

Sciences
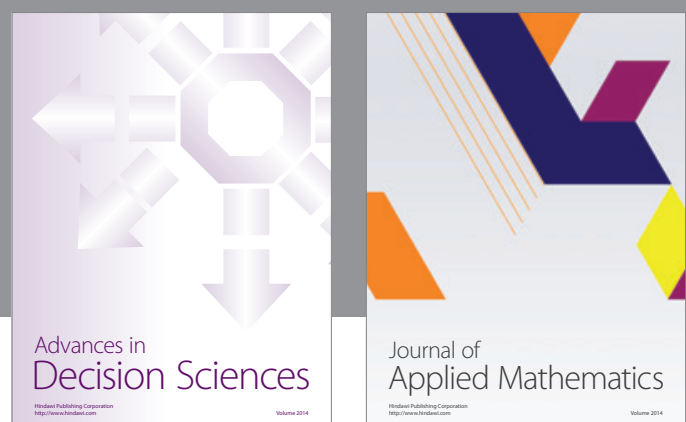

Journal of

Applied Mathematics
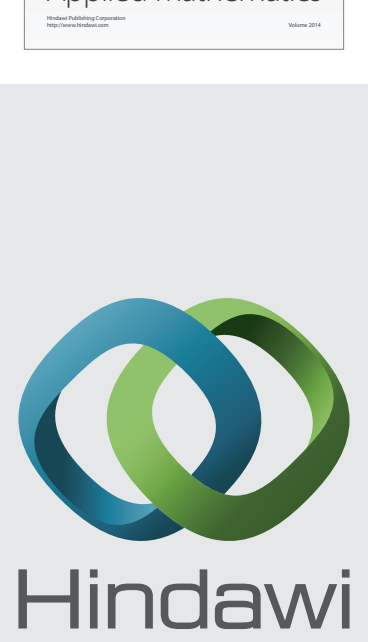

Submit your manuscripts at http://www.hindawi.com
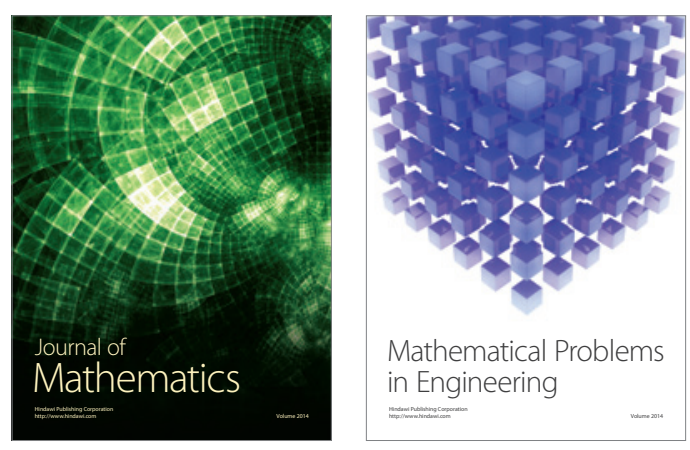

Mathematical Problems in Engineering
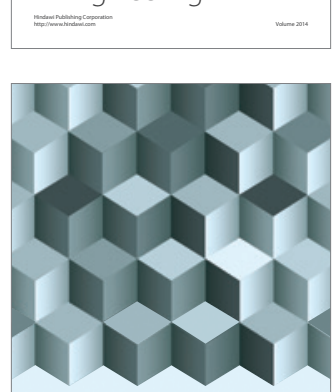

Journal of

Function Spaces
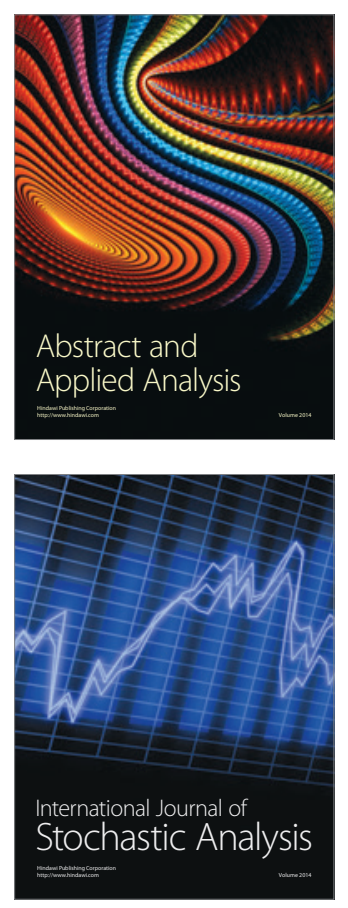

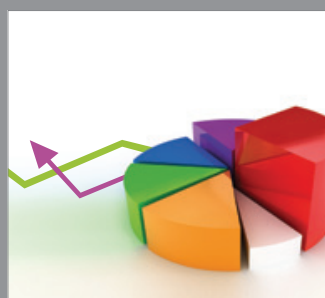

ournal of

Probability and Statistics

Promensencen
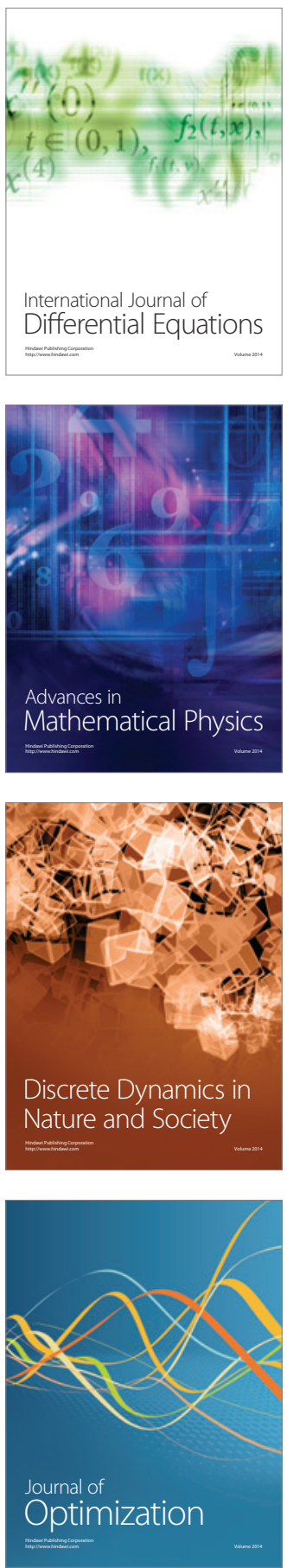